\title{
Anemia in Juvenile Idiopathic Arthritis (JIA) and Other Pediatric Rheumatologic Diseases: A Retrospective Study
}

\author{
Shatha M. Albokhari' ${ }^{1}$ Mohammed Muzaffer ${ }^{2}$ \\ ${ }^{1}$ Department of Pediatrics, Section of General Pediatrics, King Abdulaziz University, Jeddah, Saudi Arabia \\ ${ }^{2}$ Division of Rheumatology, Pediatric Department, King Abdulaziz University, Jeddah, Saudi Arabia \\ Email: halbokhari@kau.edu.sa
}

How to cite this paper: Albokhari, S.M. and Muzaffer, M. (2021) Anemia in Juvenile Idiopathic Arthritis (JIA) and Other Pediatric Rheumatologic Diseases: A Retrospective Study. Open Journal of Rheumatology and Autoimmune Diseases, 11, 188-202.

https://doi.org/10.4236/ojra.2021.114019

Received: October 5, 2021

Accepted: November 14, 2021

Published: November 17, 2021

Copyright $\odot 2021$ by author(s) and Scientific Research Publishing Inc. This work is licensed under the Creative Commons Attribution International License (CC BY 4.0).

http://creativecommons.org/licenses/by/4.0/

\section{Abstract}

Objectives: The present study estimated the prevalence of anemia among children and adolescents with pediatric rheumatological diseases in a referral center, and analyzed the associated clinical and biological parameters. $\mathbf{M e}$ thods: A retrospective chart review included 49 children with rheumatological diseases, who were diagnosed by a pediatric rheumatologist and classified according to the International League of Associations for Rheumatology (ILAR) guidelines and criteria endorsed by the American College of Rheumatologists. Anemia was defined as hemoglobin level lower than the 5th centile for the corresponding age and gender. Disease activity was indicated by serum levels of C-reactive protein (CRP) and erythrocyte sedimentation rate (ESR), where available. Results: Participants were aged 2 - 18 years (mean \pm $\mathrm{SD}=10.41 \pm 4.23$ years $), 38(77.6 \%)$ of them had JIA, and $8(16.3 \%)$ had systemic lupus erythematosus. The most frequent subtype of JIA was the polyarticular (16 out of $38,42.1 \%)$, followed by systemic $(14,36.8 \%)$. The prevalence of anemia was $46.9 \%(95 \% \mathrm{CI}=32.5 \%-61.7 \%)$, with no significant difference between JIA and other diseases or between the different JIA subtypes. Nevertheless, anemia was more frequently observed in younger patients (age 2 - 6 years: $69.2 \%$ vs $<48 \%)$ and those with elevated ESR ( $68.8 \%$ vs $33.3 \%$ ) or CRP (60.0\% versus $45.2 \%$ ), compared with their counterpart respectively; however, only the association with ESR was statistically significant ( $\mathrm{p}=$ 0.049). No agreement was found between CRP and ESR (Kappa = 0.140). Conclusion: Anemia is frequent in JIA and other rheumatologic diseases in children, concerning approximately $50 \%$ of the patients and responding to anemia of inflammation as the major pathophysiological mechanism. Further research is warranted to provide more accurate insight into the pathophysiological mechanisms and clinical characteristics of anemia in pediatric rheu- 
matological disease and to measure its morbidity, to provide efficient and evidence-based management strategies.

\section{Keywords}

Juvenile Idiopathic Arthritis, Anemia, Hemoglobin, Disease Activity, Inflammation

\section{Introduction}

Juvenile Idiopathic Arthritis (JIA) is the most frequent chronic rheumatic disorder that develops in childhood. It primarily involves joints and the diagnosis is made for all forms of arthritis that persist six weeks or longer with onset before the age of 16 years and after exclusion of any other underlying etiology. The common clinical picture includes joint inflammation, tenderness, pain, and movement limitation, with predilection for large joints [1] [2] [3]. Extra-articular manifestations such as fever, pericarditis, splenomegaly, or uveitis, are commonly reported and may precede the characteristic joint inflammation by varying lapse of time, which renders the diagnosis more challenging [1] [4]. According to the revised (2001) classification by the International League of Associations for Rheumatology (ILAR), JIA is classified into seven subtypes including oligoarticular, seropositive polyarticular, seronegative polyarticular, systemic-onset, enthesitis-related arthritis (ERA), juvenile psoriatic arthritis (JPsA) and undifferentiated JIA [5].

The incidence of JIA varies across regions and ethnic groups, ranging between 11.9 and 16.6 per 100,000 children in the United States and Europe [6] [7] [8]. In the Middle-East region, the burden of JIA is still undetermined as the epidemiological figures are not well established. The few data from Saudi Arabia, Oman and Egypt suggest a prevalence ranging from 3.43 - 20 cases per 100,000 inhabitants [4] [9] [10]. On the other hand, JIA is associated with poor prognosis and long-term outcome [11]. Besides the high risk of mortality, which was reported to be up to 50 times higher than in the general population [12], JIA morbidity is marked by several debilitating sequelae such as leg length discrepancy, muscle atrophy, joint deformities, and blindness in the uveitis forms [13] [14]. Further, extra-articular forms are associated with multiorgan complications such as pulmonary hypertension, interstitial lung disease, and myocardial injury [15] [16].

Among other systemic complications of JIA and rheumatological diseases is anemia, which constitutes the most frequent hematologic disorder of JIA [17]. The most common pathophysiological mechanism leading to anemia in JIA is inflammation, with frequently evidenced correlations with disease activity including arthritic joint count and serum levels of inflammatory markers, more specifically erythrocyte sedimentation rate (ESR) and C-reactive protein (CRP) [17] [18] [19]. Other mechanisms of anemia in rheumatological disease include iron deficiency and impaired vitamin B12 absorption, both due to gastrointes- 
tinal manifestations [20], in addition to autoimmune hemolytic anemia [21]. Hence, anemia in rheumatological diseases is multifactorial and potentially severe and may be indicative of a malignancy or a premalignancy state [22].

In the present study, we attempted to estimate the prevalence of anemia among pediatric patients with JIA and other rheumatological diseases in a referral center and to analyze its association with the diagnosis, disease severity and activity, as well as the other clinical parameters.

\section{Methods}

A retrospective chart review involved all children aged $0-18$ years who were diagnosed and followed up for JIA or other rheumatological diseases at the Pediatric Department of King Abdulaziz University Hospital, between February and October 2020. Children who had no hemoglobin results in their files were excluded. The study was performed in accordance with the ethical standards stated in the 1964 Declaration of Helsinki and its later amendments, and the study protocol was reviewed and approved by the ethical committee of King Abdulaziz University.

\subsection{Case Definition}

The diagnosis of JIA was made by a pediatric rheumatologist on a clinical picture of arthritis in one or more joints occurring for 2 weeks or more in a child aged 16 years or younger, with negative etiological investigation and after ruling out all differential diagnoses. The ILAR criteria were followed for the classification of each case as the appropriate subtype [19]. The diagnosis and classification of the other rheumatological diseases were made based on the criteria endorsed by the American College of Rheumatology (ACR), as available in their official website at:

https://www.rheumatology.org/Practice-Quality/Clinical-Support/Criteria.

\subsection{Laboratory Procedures}

Total hemoglobin concentration was measured using the $\mathrm{CBC}$ machine (SYSMEX), on blood samples collected in ethylenediaminetetraacetic acid (EDTA) tubes and in compliance with the standard procedures recommended by the manufacturer. Results are given as hemoglobin concentrations in $\mathrm{g} / \mathrm{dL}$.

CRP was measured using Alinity (ABBOTT) on blood samples collected in (NaHep) tubes and in compliance with the standard procedures recommended by the manufacturer. Results are given in $\mathrm{mg} / \mathrm{L}$.

ESR was measured using the ESR Analyzer device (BD), on blood samples collected in EDTA tubes and in compliance with the standard procedures recommended by the manufacturer. Results are given in $\mathrm{mm}$ per hour $(\mathrm{mm} / \mathrm{hr})$.

Criteria for Anemia and Inflammatory Status

Anemia was defined as a hemoglobin level lower than the 5th centile for the corresponding age and gender, that is for term newborn $(<13.5 \mathrm{~g} / \mathrm{dL}), 1$ month 
infant $(<10.7 \mathrm{~g} / \mathrm{dL}), 2$ months $(9.4 \mathrm{~g} / \mathrm{dL}), 3$ - 6 months $(<9.5 \mathrm{~g} / \mathrm{dL}), 6-24$ months $(<10.5 \mathrm{~g} / \mathrm{dL}), 2-12$ years $(<11.5 \mathrm{~g} / \mathrm{dL})$, and $12-18$ years (males: $<13$ g/dL; females: $<12 \mathrm{~g} / \mathrm{dL}$ ) [23].

Abnormal CRP results were defined as values $(\mathrm{mg} / \mathrm{L})$ higher than the 97th percentile with respect to the child's age and gender. These percentiles ranged between 3.4 - $5.5 \mathrm{mg} / \mathrm{L}$ in females and $2.6-5.3 \mathrm{mg} / \mathrm{L}$ for males [24]. Abnormal ESR was defined as level $>20 \mathrm{~mm}$ in the first hour.

\subsection{Statistical Methods}

Statistical analysis was performed with the Statistical Package for Social Sciences version 21.0 for Windows (SPSS Inc., Chicago, IL, USA). Categorical variables are presented as frequency and percentage, while continuous variables are presented as mean \pm standard deviation (SD). The agreement between CRP and ESR in indicating active inflammatory state was analyzed using Kappa coefficient. Kappa value was interpreted as no agreement $(<0)$, slight $(0-0.20)$, fair $(0.21-0.40)$, moderate $(0.41-0.60)$, substantial $(0.61-0.80)$ and perfect $(0.81$ $1.0)$ agreement [25]. A p-value of $<0.05$ was considered to reject the null hypothesis.

\section{Results}

\subsection{Participant Characteristics}

Forty-nine children were included in the study, 28 (57.1\%) were female. The mean \pm SD age was $10.41 \pm 4.23$ years (range $=2-18$ years), 13 (26.5\%) were aged 6 years or below and 19 (38.8\%) were from age group $6-12$ years. JIA represented the predominant diagnosis with $38(77.6 \%)$ cases, followed by systemic lupus erythematosus (SLE) with $8(16.3 \%)$ cases. The most common JIA subtypes were polyarticular arthritis (16 children, $42.1 \%)$ and systemic JIA (14, $36.8 \%)$, whereas the oligoarthritic subtype was present in 7 (18.5\%) children (Table 1).

\subsection{Treatment Regimens}

The majority of patients were on methotrexate (71.4\%) and or corticosteroids (61.2\%), and approximately one-half were on anti-TNF- $\alpha$ (44.9\%), while anti-IL-6R were prescribed in only $18.4 \%$. Further regimens included folic acid (44.9\%), non-steroid anti-inflammatory drugs (20.4\%) and hydroxychloroquine (14.3\%) (Table 2).

\subsection{Biological Assessments and Screening for Anemia}

Inflammatory markers showed elevation in ESR and CRP in 16 (32.7\%) and 15 (30.6\%) patients, respectively; however, ESR results were only available for 37 patients and CRP for 46 (Table 2). Analysis of hemoglobin levels with reference to age- and gender-adjusted centiles showed 23 patients with low hemoglobin, resulting in a prevalence of anemia as high as $46.9 \%(95 \% \mathrm{CI}=32.5 \%-61.7 \%)$ 
Table 1. Participants' demographic and clinical characteristics.

\begin{tabular}{|c|c|c|c|c|c|}
\hline Parameter & Category/Unit & Frequency & Percentage & Mean & SD \\
\hline \multirow{2}{*}{ Gender } & Male & 21 & 42.9 & & \\
\hline & Female & 28 & 57.1 & & \\
\hline Age & Years & & & 10.41 & 4.23 \\
\hline \multirow{3}{*}{ Category } & $2-6$ years & 13 & 26.5 & & \\
\hline & $6-12$ years & 19 & 38.8 & & \\
\hline & $12-18$ years & 17 & 34.7 & & \\
\hline \multirow{6}{*}{ Diagnosis subtype } & JIA & 38 & 77.6 & & \\
\hline & SLE & 8 & 16.3 & & \\
\hline & Other & 3 & 6.0 & & \\
\hline & Kawazaki D & 1 & 2.0 & & \\
\hline & Reactive Arthritis & 1 & 2.0 & & \\
\hline & $J D$ & 1 & 2.0 & & \\
\hline \multirow{4}{*}{ JIA subtype } & Polyarthritis & 16 & 42.1 & & \\
\hline & Systemic & 14 & 36.8 & & \\
\hline & Oligoarthritic & 7 & 18.5 & & \\
\hline & Psoriatic & 1 & 2.6 & & \\
\hline On medication ${ }^{\S}$ & Yes & 49 & 100.0 & & \\
\hline \multirow{2}{*}{ Iron therapy } & No & 31 & 63.3 & & \\
\hline & Yes & 18 & 36.7 & & \\
\hline
\end{tabular}

SD: Standard deviation; JIA: Juvenile idiopathic arthritis; SLE: Systemic lupus erythematosus; JD: Juvenile dermatomyositis; ${ }^{\complement}$ Percentages are calculated on the number of JIA cases $(\mathrm{N}=38)$.

Table 2. Treatment regimens.

\begin{tabular}{ccc}
\hline Therapeutic class/Medication & N & Percentage \\
Methotrexate & 35 & 71.4 \\
Corticosteroids (prednisone, methylprednisolone) & 30 & 61.2 \\
Anti TNF- $\alpha$ (Adalimumab, Etanercept) & 22 & 44.9 \\
Anti-IL-6R (Tocilizumab) & 9 & 18.4 \\
Hydroxychloroquine & 7 & 14.3 \\
Other immunosuppressant drug & 5 & 10.2 \\
(Mycophenolate, Cyclophosphamide) & 5 & 10.2 \\
Anti CD20 MCA (Rituximab) & 2 & 4.1 \\
IVIG & 22 & 44.9 \\
Folic acid & 10 & 20.4 \\
NSAIDs & &
\end{tabular}

MCA: Monoclonal antibody; IVIG: intravenous immunoglobulin; NSAIDs: non-steroid anti-inflammatory drugs; A patient's regimen may include more than one treatment at once. 
(Table 3). There was no agreement between ESR and CRP in the study population (Kappa $=0.140, \mathrm{p}=0.393$ ), and the agreement coefficient dropped even lower in anemic patients (Kappa $=0.024)$ (Results not presented in Tables). Further, only $3(6.1 \%)$ patients underwent testing for sickle-cell disease and 4 (8.1\%) underwent hemoglobin electrophoresis, all showing no abnormalities (Results not presented in the Tables).

\section{Clinical and Biological Factors Associated with Anemia}

There was no statistically significant difference $(\mathrm{p}=0.425)$ in the prevalence of anemia between JIA (50.0\%) and other rheumatological diseases (36.4\%). On the other hand, although not statistically significant, oligoarthritic JIA had a

Table 3. Laboratory findings.

\begin{tabular}{|c|c|c|c|c|c|c|}
\hline Parameter & $\mathbf{N}$ & Level & Freq. & $\%$ & Mean & $\mathrm{SD}$ \\
\hline \multirow{4}{*}{$\operatorname{ESR}(\mathrm{mm} / \mathrm{hr})$} & \multirow{4}{*}{37} & Mean, SD & & & 18.27 & 23.46 \\
\hline & & $\operatorname{Normal}(0-10)$ & 21 & 42.9 & & \\
\hline & & Elevated $(>10)$ & 16 & 32.7 & & \\
\hline & & Missing & 12 & 24.5 & & \\
\hline \multirow{4}{*}{$\mathrm{CRP}(\mathrm{mg} / \mathrm{mL})$} & \multirow{4}{*}{46} & Mean, SD & & & 10.58 & 19.44 \\
\hline & & Normal & 31 & 63.3 & & \\
\hline & & Elevated & 15 & 30.6 & & \\
\hline & & Missing & 3 & 6.1 & & \\
\hline \multirow{3}{*}{$\mathrm{HB}(\mathrm{g} / \mathrm{dL})$} & \multirow{3}{*}{49} & Mean, SD & & & 11.75 & 1.49 \\
\hline & & Optimal & 26 & 53.1 & & \\
\hline & & Low $\left(<5^{\text {th }}\right.$ centile $)$ & 23 & 46.9 & & \\
\hline \multirow{3}{*}{ Serum iron $(\mathrm{mcg} / \mathrm{dL})$} & \multirow{3}{*}{21} & Mean, SD & & & 12.01 & 18.12 \\
\hline & & Optimal & & & & \\
\hline & & $\operatorname{Low}(<)$ & & & & \\
\hline \multirow{2}{*}{ Serum ferritin } & \multirow{2}{*}{21} & Mean, SD & & & 380.59 & 781.74 \\
\hline & & Optimal & & & & \\
\hline \multirow{2}{*}{ TIBC } & \multirow{2}{*}{10} & Mean, SD & & & 54.32 & 14.66 \\
\hline & & Optimal & & & & \\
\hline \multirow{2}{*}{ Liver function test } & \multirow{2}{*}{49} & Normal & 48 & 98.0 & & \\
\hline & & Abnormal & 1 & 2.0 & & \\
\hline \multirow{2}{*}{ Renal function test } & & Normal & 48 & 98.0 & & \\
\hline & & Abnormal & 1 & 2.0 & & \\
\hline
\end{tabular}

SD: Standard deviation; ESR: Erythrocyte sedimentation rate; CRP: C-reactive protein; TIBC: Total iron binding capacity. 
relatively higher prevalence of anemia (71.4\%) compared with the other JIA subtypes (up to $50 \%, \mathrm{p}=0.469$ ). Of note, we observed a higher prevalence of anemia among younger children, accounting for 9/13 (69.2\%) of them, compared with $31.6 \%$ and $47.1 \%$ in the older age groups $6-12$ years and $12-18$ years, respectively; however, the difference was not statistically significant. No gender effect was observed $(p=0.934)$. Biologically, the prevalence of anemia was significantly higher among patients with high ESR (68.8\%) compared with their counterparts $(33.3 \%),(p=0.049)$. Although no association of anemia was observed with CRP $(p=0.345)$, the combination of the two markers showed a higher prevalence of anemia in case that both markers were elevated $(71.4 \%)$ versus one (58.8\%) and none (34.8\%); however, the result did not reach statistical significance $(\mathrm{p}=0.140)$. Measurement of serum ferritin levels was available for 13 out of the 23 anemic patients, and was normal (9/13) or high (4/13) in all cases indicating anemia of inflammation. Of note, only 12 out of 23 patients with anemia were on iron therapy (Table 4). No association was otherwise found between anemia and the treatment regimen (Table 5). Pearson's correlation test showed significant negative relationship of hemoglobin levels with ESR ( $r=$ $-0.559, \mathrm{p}<0.001)$ and non-significant relationship with CRP $(\mathrm{r}=-0.128, \mathrm{p}=$ 0.398) (Results are not presented in Tables).

\section{Discussion}

\subsection{Summary of Findings}

This retrospective study showed that anemia is common among children with rheumatological diseases, accounting for approximately 50\% in JIA and 36.4\% for other diseases, and its prevalence was likely to be decreasing with age. Anemia of inflammation was likely to be the major mechanism of anemia, and the prevalence of anemia was significantly associated with elevated ESR levels, while it was not associated with CRP level. The combination of the two markers showed even higher frequency of anemia (71.4\%) in case of elevation of both markers; however, this was not statistically significant. Further, children with oligoarthritic subtype were likely to have a higher prevalence of anemia. On the other hand, findings from this chart review suggest that approximately $50 \%$ of anemia cases did not benefit from the appropriate management. The discussion section will focus on JIA as the most prevalent diagnosis in our study sample.

\subsection{Anemia in Children with JIA and Other Rheumatological Diseases: Prevalence and Mechanisms}

Anemia is a frequent systemic complication in children with JIA or other rheumatological diseases and may respond to different pathological mechanisms. The most common pathophysiologic mechanism is anemia of inflammation. It is more frequently reported in systemic-onset JIA, where it is usually microcytic, due to defect in iron supply for erythropoiesis [25] [26] [27]. However, this defect is usually due to iron accumulation in the reticuloendothelial system and is often 
Table 4. Clinical and biological factors associated with anemia.

\begin{tabular}{|c|c|c|c|c|c|c|}
\hline \multirow[t]{2}{*}{ Factor } & \multirow{2}{*}{$\begin{array}{c}\text { Category } \\
2-6\end{array}$} & \multicolumn{2}{|c|}{$\begin{array}{c}\text { No anemia } \\
(N=26)\end{array}$} & \multicolumn{2}{|c|}{$\begin{array}{l}\text { Anemia } \\
(\mathrm{N}=23)\end{array}$} & \multirow[t]{2}{*}{ p-value } \\
\hline & & 4 & 30.8 & 9 & 69.2 & \\
\hline \multirow[t]{2}{*}{ Age (years) } & $6-12$ & 13 & 65.8 & 6 & 31.6 & \\
\hline & $12-18$ & 9 & 52.9 & 8 & 47.1 & 0.111 \\
\hline \multirow{2}{*}{ Gender } & Male & 11 & 52.4 & 10 & 47.6 & \\
\hline & Female & 15 & 53.6 & 13 & 46.4 & 0.934 \\
\hline \multirow{5}{*}{ Diagnosis } & JIA & 19 & 50.0 & 19 & 50.0 & \\
\hline & SLE & 5 & 62.5 & 3 & 37.5 & \\
\hline & Kawazaki D & 0 & 0.0 & 1 & 100.0 & \\
\hline & Reactive Arthritis & 1 & 100.0 & 0 & 0.0 & \\
\hline & JD & 1 & 100.0 & 0 & 0.0 & 0.504 \\
\hline \multirow{2}{*}{ JIA vs others } & JIA & 19 & 50.0 & 19 & 50.0 & \\
\hline & Other diseases & 7 & 63.6 & 4 & 36.4 & $0.425^{\mathrm{F}}$ \\
\hline \multirow{4}{*}{ JIA Subtype ${ }^{\S}$} & Polyarthritic & 9 & 56.3 & 7 & 43.8 & \\
\hline & Systemic & 7 & 50.0 & 7 & 50.0 & \\
\hline & Oligoarthritic & 2 & 28.6 & 5 & 71.4 & \\
\hline & Psoriatic & 1 & 100.0 & 0 & 0.0 & 0.469 \\
\hline \multirow{2}{*}{ Systemic JIA ${ }^{\S}$} & Systemic & 7 & 50.0 & 7 & 50.0 & \\
\hline & Others & 12 & 50.0 & 12 & 50.0 & $1.000^{\mathrm{F}}$ \\
\hline \multirow{2}{*}{ ESR } & Normal & 14 & 66.7 & 7 & 33.3 & \\
\hline & Elevated & 5 & 31.3 & 11 & 68.8 & $0.049^{\star \mathrm{F}}$ \\
\hline \multirow{2}{*}{ CRP } & Normal & 17 & 54.8 & 14 & 45.2 & \\
\hline & Elevated & 6 & 40.0 & 9 & 60.0 & 0.345 \\
\hline \multirow{3}{*}{ ESR or CRP } & Both normal & 15 & 65.2 & 8 & 34.8 & \\
\hline & One elevated & 7 & 41.2 & 10 & 58.8 & \\
\hline & Both elevated & 2 & 2.6 & 5 & 71.4 & 0.140 \\
\hline \multirow{2}{*}{ Iron therapy } & No & 20 & 64.5 & 11 & 35.5 & \\
\hline & Yes & 6 & 33.3 & 12 & 66.7 & $0.035^{\star}$ \\
\hline \multirow{3}{*}{ Serum ferritin } & Low & 1 & 100.0 & 0 & 0.0 & \\
\hline & Optimal $(7-140)$ & 4 & 30.8 & 9 & 69.2 & \\
\hline & High & 3 & 42.9 & 4 & 57.1 & 0.370 \\
\hline
\end{tabular}

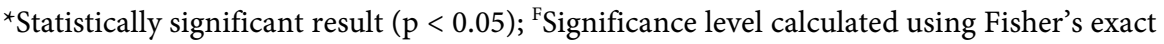
test; otherwise, chi-square test was used; ${ }^{5}$ Percentages are calculated on the corresponding number of JIA cases, by exclusion of other diagnoses. 
Table 5. Association of anemia with treatment regimen.

\begin{tabular}{|c|c|c|c|c|}
\hline \multirow{3}{*}{$\begin{array}{c}\text { Therapeutic class/Medication } \\
\text { Methotrexate }\end{array}$} & \multirow{3}{*}{$\begin{array}{c}\text { Category } \\
\text { No } \\
\text { Yes }\end{array}$} & \multicolumn{2}{|c|}{ Anemia $(\mathrm{N}, \%)$} & \multirow{3}{*}{$\begin{array}{c}\text { p-value } \\
0.717\end{array}$} \\
\hline & & 6 & 42.9 & \\
\hline & & 17 & 48.6 & \\
\hline \multirow{2}{*}{ Corticosteroids } & No & 9 & 47.4 & \\
\hline & Yes & 14 & 46.7 & 0.962 \\
\hline \multirow{2}{*}{ Anti TNF- $\alpha$} & No & 15 & 55.6 & \\
\hline & Yes & 8 & 36.4 & 0.181 \\
\hline \multirow{2}{*}{ Anti-IL-6R } & No & 21 & 52.5 & \\
\hline & Yes & 2 & 22.2 & $0.145^{\mathrm{F}}$ \\
\hline \multirow{2}{*}{ Hydroxychloroquine } & No & 20 & 47.6 & \\
\hline & Yes & 3 & 42.9 & $1.000^{\mathrm{F}}$ \\
\hline \multirow{2}{*}{ Other immunosuppressant drugs } & No & 22 & 50.0 & \\
\hline & Yes & 1 & 20.0 & $0.353^{\mathrm{F}}$ \\
\hline \multirow{2}{*}{ Anti CD20 MCA } & No & 21 & 47.7 & \\
\hline & Yes & 2 & 40.0 & $1.000^{\mathrm{F}}$ \\
\hline \multirow{2}{*}{ Folic acid } & No & 12 & 44.4 & \\
\hline & Yes & 11 & 50.0 & 0.698 \\
\hline \multirow{2}{*}{ NSAIDs } & No & 16 & 41.0 & \\
\hline & Yes & 7 & 70.0 & $0.157^{\mathrm{F}}$ \\
\hline
\end{tabular}

MCA: Monoclonal antibody; IVIG: intravenous immunoglobulin; NSAIDs: non-steroid anti-inflammatory drugs; A patient's regimen may include more than one treatment at once. Fignificance level calculated using Fisher's exact test; otherwise, chi-square test was used.

associated with increased serum transferrin receptor (TfR). These abnormalities are believed to be the effect of increased TNF- $\alpha$, which has an inhibitory action on erythropoiesis and may cause apoptosis of bone marrow erythroid cells in the long term. A study showed that anti-TNF therapy improved iron status of anemic JIA children, as it restored the hemoglobin levels and mean corpuscular volume of red blood cells (MCV) [28].

In JIA, anemia occurs often during disease onset or exacerbation, and sometimes in conjunction with other more severe extra-articular manifestations such as pericarditis and myocarditis [29]. Among the other mechanisms by which JIA is suspected to induce chronic anemia in JIA is the frequent presence of subclinical gastrointestinal lesions, notably due to Helicobacter pylori, which could only be evidenced in endoscopy [30]. Other cases of anemia in JIA may be indicative of malignancy, which suggests that the highest attention should be given to anemic patients in order to address the etiology [22]. Therefore, anemia among JIA patients should be promptly recognized and properly managed. 
In the present study, anemia was found in $47 \%$ of children with rheumatic diseases, all of them were likely not in exacerbation phase. By focusing on JIA, although not statistically significant, the prevalence of anemia was relatively higher in patients with oligoarthritic subtype compared to those with other subtypes including systemic. This may be due to patients with systemic JIA being more often treated for anemia. In 2015, a previous study conducted in our center, and coauthored by the two authors of the present study, anemia was found in 59.8\% among a cohort of 82 JIA children, representing the most frequent biological abnormality. However, the study did not analyze the correlation of anemia with disease subtype or activity [19]. Internationally, a study from Thailand explored the hematological characteristics of 55 newly diagnosed JIA children and reported even higher percentage (83.6\%) of anemia cases, $86.9 \%$ of which were anemia of inflammation and $8.6 \%$ required transfusion. The same study showed that children with systemic JIA were more exposed to anemia and had lower levels of hemoglobin than other subtypes [17]. By comparison, this extremely high prevalence of anemia in newly diagnosed JIA children may support the relatively higher prevalence found in younger children that were found in our study, which may correspond to an early phase of the disease confounded by the patient's age. Altogether, these data are concordant with regards to the high prevalence of anemia of inflammation in JIA and other rheumatological diseases; however, further research is warranted to explore its pathophysiological and clinical characteristics, along with its prognostic value and determine the evidence-based management strategies.

\subsection{Inflammatory Markers, Disease Activity and Anemia}

The evaluation of patients with rheumatological diseases requires differentiating active from inactive state of the disease, as these diseases are characterized by flare-ups and remission phases. Regular assessment of disease activity is a crucial step of the clinical assessment of afflicted children, as it provides key indications on the functional prognosis, efficacy of the antirheumatic drugs and patient's well-being. In case of JIA, several criteria have been proposed to provide an accurate estimate and enable overtime monitoring of the level of disease activity in a given patient. Earlier, in 2009, the Juvenile Arthritis Disease Activity Score (JADAS) used four criteria: 3 clinical and one biological criterion. The clinical criteria included the physician's global assessment and patient/parent's global scoring of well-being, both using a 0 - 10 scale, as well as the active joint count. The biological parameter consisted of ESR level, which was normalized to a 0 10 scale. The JADAS was proposed in three versions, JADAS-71, JADAS-27 and JADAS-10, which differed by the number of joints to be assessed. All of the versions were demonstrated to be valid in discriminating disease activity and adapted for clinical use. However, according to the developing team, the use of ESR instead of CRP was motivated by its availability in the data sets where the score was validated [31] [32]. Subsequently, in 2011, a Nordic team has tested the validity of using CRP as an alternative to ESR in JADAS and found excellent 
correlation of the modified score with the original one $(r=0.99)$, in addition to comparable validity, discriminative ability and sensitivity to change [33]. However, a study by Mcerlane et al. evaluated the validity of using only the 3 clinical criteria of the JADAS, with exclusion of the ESR. By applying the three modified versions of JADAS on 352 newly diagnosed JIA children with different ILAR subtypes of the disease, authors observed improved construct validity with respect of the three clinical criteria, while ESR did not correlate well with the original or modified score. Authors suggested that the exclusion of the laboratory criterion, ESR, would improve the feasibility and clinical applicability of the scale [34]. On the other hand, the American College of Rheumatology (ACR) recommendations adopted, in 2011, a more simplified algorithm using the same criteria of JADAS, thereby stratifying JIA disease activity into three levels including low, moderate and high disease activity. Thus, the ACR used ESR but not CRP, in addition to the three other clinical criteria [35]. Other studies demonstrated that CRP and ESR levels, as well IgA rheumatoid factor, were significant indicators of JIA disease activity, all being associated with active synovitis, while other biomarkers such as anti-cyclic citrullinated peptide (anti-CCP) antibodies and cartilage oligomeric matrix protein (COMP) were associated with joint damage [36]. These observations support the interchangeable use of CRP and ESR in measuring the disease severity using composite scores.

In the present study, we observed a significant, two-fold increase in the prevalence of anemia in case of elevation of ESR, while elevated CRP was associated with non-significant $25 \%$ increase in anemia. However, in absence of the clinical criteria of disease activity, which constitutes a limitation for this study, the elevation of ESR should be interpreted with caution in the context of anemia. Indeed, anemia constitutes a factor that increases ESR owing to the fact that reduced hematocrit alters the upward flow of blood plasma resulting in faster aggregation of erythrocytes [37]. Thus, the reliability of using ESR as the sole indicator of disease activity is highly questionable in case of anemia. This suggests that the significant association of anemia with ESR that was found in the present study is more probably due to ESR being caused by anemia. On the other hand, when combining ESR with CRP, we observed a substantial increase in the prevalence of anemia when both markers were elevated. Nonetheless, the latter finding was not statistically significant, probably due to the small sample size inducing type II error and reducing the power of the subgroup analysis. On the other hand, there was no agreement between ESR and CRP in indicating inflammation in the present cohort, and the agreement coefficient Kappa dropped from 0.140 to 0.024 in anemic patients with reference to the total study population. As a consequence, the validity of using ESR in composite scores measuring JIA disease activity, in case of anemia, should be further analyzed and may be substituted by CRP or other reliable inflammatory biomarkers.

\section{Limitations}

This study has some limitations that may impede the interpretation and genera- 
lizability of the results. The analysis of the correlation of anemia with disease activity was made difficult by the absence of clinical criteria enabling the calculation of severity and disease activity, such as the JADAS score. The significantly increased ESR level in anemic patients is probably the effect of anemia rather than being the effect of inflammation. Missing data about serum ferritin in approximately $50 \%$ of anemic patients hindered the exploration of the anemia mechanism. As such, the absence of data on hemoglobin electrophoresis in the majority of patients may also constitute a limitation in a population with a relatively high incidence of hemoglobinopathies. Other factors of anemia were not available such as disease duration, which would provide further insight into the issue.

\section{Conclusion}

Anemia is frequent among children with JIA and other rheumatological diseases, concerning approximately $50 \%$ of the patients and responding to anemia of inflammation as the major pathophysiological mechanism. It is likely to be more frequent at younger age and or in the early phase of the disease, as well as during disease exacerbations. The use of ESR levels in composite scoring methods measuring disease activity should be reviewed in anemic patients, given the independent association of anemia with high ESR levels. A scoring system for JIA activity including CRP or other inflammation biomarkers should be explored. Further research is warranted to provide more accurate insight into the pathophysiological mechanisms and clinical characteristics of anemia in JIA and other rheumatological diseases, measure its morbidity and provide efficient and evidence-based management strategies.

\section{Conflicts of Interest}

The authors declare no conflicts of interest regarding the publication of this paper.

\section{References}

[1] Hahn, Y.S. and Kim, J.G. (2010) Pathogenesis and Clinical Manifestations of Juvenile Rheumatoid Arthritis. Korean Journal of Pediatrics, 53, 921-930. https://doi.org/10.3345/kjp.2010.53.11.921

[2] Barut, K., Adrovic, A., Şahin, S. and Kasapçopur, Ö. (2017) Juvenile Idiopathic Arthritis. Balkan Medical Journal, 34, 90-101. https://doi.org/10.4274/balkanmedj.2017.0111

[3] Giancane, G., Consolaro, A., Lanni, S., Davì, S., Schiappapietra, B. and Ravelli, A. (2016) Juvenile Idiopathic Arthritis: Diagnosis and Treatment. Rheumatology and Therapy, 3, 187-207. https://doi.org/10.1007/s40744-016-0040-4

[4] Abou El-Soud, A.M., El-Najjar, A.R., El-Shahawy, E.E., Amar, H.A., Hassan, T.H., Abd-Allaha, S.H., et al. (2013) Prevalence of Juvenile Idiopathic Arthritis in Sharkia Governorate, Egypt: Epidemiological Study. Rheumatology International, 33, 2315-2322. https://doi.org/10.1007/s00296-013-2707-2

[5] Cassidy, J.T. and Petty, R.E. (2005) Chronic Arthritis in Childhood. In: Cassidy, 
J.T., Petty, R.E., Lindsley, C.B., et al., Eds., Textbook of Pediatric Rheumatology, 5th Edition, Saunders, Philadelphia, 206-260.

https://doi.org/10.1016/B978-1-4160-0246-8.50015-2

[6] Harrold, L.R., Salman, C., Shoor, S., Curtis, J.R., Asgari, M.M., Gelfand, J.M., et al. (2013) Incidence and Prevalence of Juvenile Idiopathic Arthritis among Children in a Managed Care Population, 1996-2009. The Journal of Rheumatology, 40, 1218-1225. https://doi.org/10.3899/jrheum.120661

[7] Berthold, E., Månsson, B. and Kahn, R. (2019) Outcome in Juvenile Idiopathic Arthritis: A Population-Based Study from Sweden. Arthritis Research \& Therapy, 21, Article No. 218. https://doi.org/10.1186/s13075-019-1994-8

[8] Thomschke, S., Schulz, M. and Bätzing, J. (2018) Epidemiology and Health Services of Juvenile Idiopathic Arthritis (JIA) in Germany-An Analysis Based on Nationwide SHI-Physicians Claims Data from 2009 to 2015. Report No. 1, Central Research Institute for Ambulatory Health Care in Germany, Berlin.

[9] Abdwani, R., Abdalla, E., Al Abrawi, S. and Al-Zakwani, I. (2015) Epidemiology of Juvenile Idiopathic Arthritis in Oman. Pediatric Rheumatology Online Journal, 13, Article No. 33. https://doi.org/10.1186/s12969-015-0030-Z

[10] Bahabri, S., Al-Sewairi, W., Al-Mazyad, A., Karrar, A., Al-Ballaa, S., El-Ramahai, K., et al. (1997) Juvenile Rheumatoid Arthritis: The Saudi Experience. Annals of Saudi Medicine, 17, 413-418. https://doi.org/10.5144/0256-4947.1997.413

[11] Huang J.L. (2012) New Advances in Juvenile Idiopathic Arthritis. Chang Gung Medical Journal, 35, 1-14. https://doi.org/10.4103/2319-4170.106171

[12] Davies, R., Southwood, T., Kearsley-Fleet, L., Lunt, M., Baildam, E., Beresford, M.W., et al. (2017) Mortality Rates Are Increased in Patients with Systemic Juvenile Idiopathic Arthritis. Archives of Disease in Childhood, 102, 206-207. https://doi.org/10.1136/archdischild-2016-311571

[13] de Oliveira Sato, J., Corrente, J.E. and Saad Magalhães, C. (2011) Progression of Articular and Extraarticular Damage in Oligoarticular Juvenile Idiopathic Arthritis. Clinical and Experimental Rheumatology, 29, 871-877.

[14] Abu Samra, K., Maghsoudlou, A. and Roohipoor, R. (2016) Current Treatment Modalities of JIA-Associated Uveitis and Its Complications: Literature Review. Ocular Immunology and Inflammation, 24, 431-439.

https://doi.org/10.3109/09273948.2015.1115878

[15] Kimura, Y., Weiss, J.E., Haroldson, K.L., Lee, T., Punaro, M., Oliveira, S., et al. (2013) Pulmonary Hypertension and Other Potentially Fatal Pulmonary Complications in Systemic Juvenile Idiopathic Arthritis. Arthritis Care \& Research, 65, 745-752. https://doi.org/10.1002/acr.21889

[16] Abdel Massih, A.F., Salem, A., Arabi, S., Malak, L. and Marzouk, H. (2019) Intensity of Inflammation as the Most Important Predictor of Myocardial Involvement in JIA. A 3D Echocardiographic Study. Acta Reumatologica Portuguesa, 44, 296-302.

[17] Buaboonnam, J. and Charuvanij, S. (2017) Hematologic Involvement in Thai Patients with Juvenile Idiopathic Arthritis. Southeast Asian Journal of Tropical Medicine and Public Health, 48, 141-150.

[18] Vatutin, M. (2014) Differential Diagnosis of Anemia in Patients with Rheumatoid Arthritis. Archives of Rheumatology, 29, 42-46.

https://doi.org/10.5606/tjr.2014.3538

[19] Al-Hemairi, M.H., Albokhari, S.M. and Muzaffer, M.A. (2016) The Pattern of Juvenile Idiopathic Arthritis in a Single Tertiary Center in Saudi Arabia. International Journal of Inflammation, 2016, Article ID: 7802957. 
https://doi.org/10.1155/2016/7802957

[20] Kröner P.T., Tolaymat O.A., Bowman A.W., Abril, A. and Lacy, B.E. (2019) Gastrointestinal Manifestations of Rheumatological Diseases. Official Journal of the American College of Gastroenterology, 114, 1441-1454.

https://doi.org/10.14309/ajg.0000000000000260

[21] Gormezano, N.W.S., Kern, D., Pereira, O.L., Esteves, G.C.X., Sallum, A.M.E., Aikawa, N.E., et al. (2017) Autoimmune Hemolytic Anemia in Systemic Lupus Erythematosus at Diagnosis: Differences between Pediatric and Adult Patients. Lupus, 26, 426-430. https://doi.org/10.1177/0961203316676379

[22] Bloxham, E., Vagadia, V., Scott, K., Francis, G., Saravanan, V., Heycock, C., et al. (2011) Anaemia in Rheumatoid Arthritis: Can We Afford to Ignore It? Postgraduate Medical Journal, 87, 596-600. https://doi.org/10.1136/pgmj.2011.117507

[23] Wang, M. (2016) Iron Deficiency and Other Types of Anemia in Infants and Children. American Family Physician, 93, 270-278.

[24] Schlenz, H., Intemann, T., Wolters, M., González-Gil, E.M., Nappo, A., Fraterman, A., et al. (2014) C-Reactive Protein Reference Percentiles among Pre-Adolescent Children in Europe Based on the IDEFICS Study Population. International Journal of Obesity, 38, S26-S31. https://doi.org/10.1038/ijo.2014.132

[25] Landis, J.R. and Koch, G.G. (1977) The measurement of observer agreement for categorical data. Biometrics, 33, 159-174. https://doi.org/10.2307/2529310

[26] Martini, A. (2012) Systemic Juvenile Idiopathic Arthritis. Autoimmunity Reviews, 12, 56-59. https://doi.org/10.1016/j.autrev.2012.07.022

[27] Bruck, N., Schnabel, A. and Hedrich, C.M. (2015) Current Understanding of the Pathophysiology of Systemic Juvenile Idiopathic Arthritis (sJIA) and Target-Directed Therapeutic Approaches. Clinical Immunology, 159, 72-83. https://doi.org/10.1016/j.clim.2015.04.018

[28] Aalto, K., Honkanen, V. and Lahdenne, P. (2011) Iron Status during Anti-TNF Therapy in Children with Juvenile Idiopathic Arthritis. Clinical Rheumatology, 30, 115-119. https://doi.org/10.1007/s10067-010-1571-7

[29] Ravelli, A., Schiappapietra, B., Verazza, S. and Martini, A. (2017) Juvenile Idiopathic Arthritis. In: Nussinovitch, U., Ed., The Heart in Rheumatic, Autoimmune and Inflammatory Diseases, Academic Press, Cambridge, 167-187. https://doi.org/10.1016/B978-0-12-803267-1.00007-7

[30] Len, C., Hilario, M.O., Kawakami, E., et al. (1999) Gastroduodenal Lesions in Children with Juvenile Rheumatoid Arthritis. Hepato-Gastroenterology, 46, 991-996.

[31] Consolaro, A., Ruperto, N., Bazso, A., Pistorio, A., Magni-Manzoni, S., Filocamo, G., et al. (2009) Development and Validation of a Composite Disease Activity Score for Juvenile Idiopathic Arthritis. Arthritis and Rheumatism, 61, 658-666.

https://doi.org/10.1002/art.24516

[32] Consolaro, A., Bracciolini, G., Ruperto, N., Pistorio, A., Magni-Manzoni, S., Malattia, C., et al. (2012) Remission, Minimal Disease Activity, and Acceptable Symptom State in Juvenile Idiopathic Arthritis: Defining Criteria Based on the Juvenile Arthritis Disease Activity Score. Arthritis and Rheumatism, 64, 2366-2374.

https://doi.org/10.1002/art.34373

[33] Nordal, E.B., Zak, M., Aalto, K., Berntson, L., Fasth, A., Herlin, T., et al. (2012) Validity and Predictive Ability of the Juvenile Arthritis Disease Activity Score Based on CRP versus ESR in a Nordic Population-Based Setting. Annals of the Rheumatic Diseases, 71, 1122-1127. https://doi.org/10.1136/annrheumdis-2011-200237

[34] McErlane, F., Beresford, M.W., Baildam, E.M., Chieng, S.E.A., Davidson, J.E., Fos- 
ter, H.E., et al. (2013) Validity of a Three-Variable Juvenile Arthritis Disease Activity Score in Children with New-Onset Juvenile Idiopathic Arthritis. Annals of the Rheumatic Diseases, 72, 1983-1988.

https://doi.org/10.1136/annrheumdis-2012-202031

[35] Beukelman, T., Patkar, N.M., Saag, K.G., Tolleson-Rinehart, S., Cron, R.Q., DeWitt, E.M., et al. (2011) 2011 American College of Rheumatology Recommendations for the Treatment of Juvenile Idiopathic Arthritis: Initiation and Safety Monitoring of Therapeutic Agents for the Treatment of Arthritis and Systemic Features. Arthritis Care \& Research, 63, 465-482. https://doi.org/10.1002/acr.20460

[36] Gilliam, B.E., Chauhan, A.K., Low, J.M. and Moore, T.L. (2008) Measurement of Biomarkers in Juvenile Idiopathic Arthritis Patients and their Significant Association with Disease Severity: A Comparative Study. Clinical and Experimental Rheumatology, 26, 492-497.

[37] Brigden, M.L. (1999) Clinical utility of the erythrocyte sedimentation rate. American Family Physician, 60, 1443-1450.

https://www.aafp.org/afp/1999/1001/p1443.html 